\title{
Progressão do cateter central de inserção periférica em região hemiclavicular de recém-nascidos*
}

\author{
Progression of peripherally inserted central catheter in hemiclavicular region of newborns
}

\section{Como citar este artigo:}

Nobre KSS, Cardoso MVLML, Rodrigues EC, Melo GM. Progression of peripherally inserted central catheter in hemiclavicular region of newborns. Rev Rene. 2020;21:e42980. DOI: https://doi.org/10.15253/2175-6783.20202142980

Keline Soraya Santana Nobre ${ }^{1}$

(D)Maria Vera Lúcia Moreira Leitão Cardoso ${ }^{1}$

(DElisa da Conceição Rodrigues ${ }^{2}$

(D)Gleicia Martins de Melo ${ }^{1}$

\author{
*Extraído da Dissertação de Mestrado "Manobra de \\ movimentação do ombro para progressão do cateter \\ central de inserção periférica em unidade neonatal", \\ Universidade Federal do Ceará, 2014. \\ ${ }^{1}$ Universidade Federal do Ceará. \\ Fortaleza, CE, Brasil. \\ ${ }^{2}$ Universidade Federal do Rio de Janeiro. \\ Rio de Janeiro, RJ, Brasil.

\section{Autor correspondente:} \\ Maria Vera Lúcia Moreira Leitão Cardoso \\ Rua Alexandre Baraúna, 1115, Rodolfo Teófilo, \\ CEP: 60430-160, Fortaleza, CE, Brasil. \\ E-mail: cardoso@ufc.br
}

\section{RESUMO}

Objetivo: avaliar a progressão do cateter central de inserção periférica em região hemiclavicular direita, através da veia basílica e cefálica direita, em recém-nascidos. Métodos: pesquisa quase experimental, realizada em unidade neonatal. Amostra de 64 inserções de cateteres, em 58 recém-nascidos. A intervenção consistiu em manobra de elevação, protração e abaixamento do ombro, aplicada após não progressão do cateter, em região hemiclavicular, na punção direta em região cubital, em veia basílica ou cefálica direita. Resultados: das 64 inserções, progrediram sem manobra 28(43,7\%); em mais da metade, aplicou-se manobra, obtendo-se $28(77,8 \%)$ progressões, sendo que $15(41,7 \%)$ progrediram após elevação, $12(57,1 \%)$ após protração e $1(11,1 \%)$ abaixamento do ombro, destes que progrediram, $21(75 \%)$ estavam em posição central. Verificou-se significância estatística $(p<0,05)$ entre progressão com manobra e veia cefálica, progressão sem manobra e veia basílica. Conclusão: a intervenção facilitou progressão do cateter, principalmente por veia cefálica.

Descritores: Cateterismo Venoso Central; Cateterismo Periférico; Recém-Nascido; Infusões Intravenosas; Enfermagem Neonatal.

\section{ABSTRACT}

Objective: to evaluate the progression of the peripherally inserted central catheter in the right hemiclavicular region, through the right basilic and cephalic vein, in newborns. Methods: quasi-experimental research, carried out in a neonatal unit. Sample of 64 catheter insertions in 58 newborns. The intervention consisted of shoulder elevation, protraction and lowering maneuver, applied after the catheter had not progressed, in the hemiclavicular region by direct puncture in the cubital region, in the right basilic or cephalic vein. Results: of the 64 insertions, 28(43.7\%) progressed without maneuver; in more than half, a maneuver was applied, obtaining $28(77.8 \%)$ progressions, with $15(41.7 \%)$ progressing after elevation, 12(57.1\%) after protraction, $1(11.1 \%)$ lowering the shoulder, of those that progressed, $21(75 \%)$ were in central position. There was statistical significance $(\mathrm{p}<0.05)$ between progression with maneuver and cephalic vein, progression without maneuver and basilic vein. Conclusion: the intervention facilitated the progression of the catheter, mainly through the cephalic vein.

Descriptors: Catheterization, Central Venous; Catheterization, Peripheral; Infant, Newborn; Infusions, Intravenous; Neonatal Nursing. 


\section{Introdução}

Cateter Central de Inserção Periférica (PICC) é um dispositivo menos invasivo quando comparado com cateter inserido por punção venosa central, podendo ser introduzido por veias periféricas do couro cabeludo (temporal e auricular posterior), membros inferiores (safena) e superiores (basílica, cefálica e axilar) em recém-nascidos ${ }^{(1)}$.

A inserção do PICC em neonatologia pode ser feita através da punção direta ou técnica de Seldinger modificada e ultrassom ${ }^{(2)}$. Durante a prática da inserção do cateter, observa-se, em alguns casos, a não progressão pelas veias basílica e cefálica, podendo estar associado à estenose, veias tortuosas, venoespasmo, bifurcações e válvulas venosas fechadas, tromboses, hematomas, calibre desproporcional entre vaso e cateter $^{(3)}$.

Para superar a dificuldade de progressão do PICC pela veia, sugere-se infusão de soro fisiológico em bolus, massagem suave na veia na direção do fluxo de sangue, compressa morna, promovendo dilatação, reposicionamento e/ou rotação do membro ${ }^{(4)}$, porém, até o presente momento, a literatura não relata detalhamento dessa rotação, nem quanto facilita a progressão do cateter até veia cava superior.

Contudo, constata-se não progressão na região hemiclavicular direita, próximo ao ombro, pela cateterização das veias basílica e cefálica em recém-nascidos, a qual pode estar relacionada à angulação de $90^{\circ}$ da implantação de veia cefálica em veia axilar, ao penetrar na fáscia clavipeitoral, passando sob a clavícu$\mathrm{la}^{(3)}$ e possibilidade de compressão da veia subclávia pela clavícula e primeira costela, no ângulo costoclavicular estreitado, sendo obstáculos à progressão, como ocorre na Síndrome de Pinch-off ${ }^{5)}$. A má progressão do cateter pode promover falso trajeto e posicionamento inadequado e este, por sua vez, está associado à oclusão do vaso, sendo a migração e oclusão do cateter as mais comuns complicações com PICC em neonatos ${ }^{(6)}$. Diante disso, reconheceu-se a necessidade de criar manobra que pudesse favorecer a progressão do
PICC na região hemiclavicular direita.

A manobra de movimentação do ombro para progressão do PICC em recém-nascidos, estudada neste artigo, foi criada pelas duas primeiras autoras, é descrita em três passos, para aumentar o espaço da subclávia entre primeira costela e clavícula, diminuindo a compressão, favorecendo progressão, como segue: elevação do ombro (aplicação de leve pressão na região axilar, no sentido podocefálico); protração do ombro (leve pressão na região escapular no sentido posteroanterior, com deslocamento do ombro para frente); abaixamento do ombro (aplicação de leve pressão sobre o ombro do recém-nascido, deslocando-o para baixo, no sentido cefalopodal, com deslocamento do ombro para baixo).

Boas práticas inovadoras no cuidado de enfermagem na inserção de PICC em recém-nascidos favorecem o sucesso na inserção, mitigando insucesso nas inserções, perda de cateter e exposição desnecessária do recém-nascido a maior tempo de procedimento, punções venosas repetidas e risco de posicionamento inadequado.

Com isso, investigou-se a progressão do PICC, na região hemiclavicular direita, pela inserção em veia basílica ou cefálica direita, da região cubital de recém-nascidos, com e sem aplicação da manobra. Objetivou-se avaliar a progressão do cateter central de inserção periférica em região hemiclavicular direita, através da veia basílica e cefálica direita, em recém-nascidos.

\section{Métodos}

Pesquisa quase experimental, com desenho pré e pós-teste em um só grupo, que consiste na aplicação de pré-teste (prévio à intervenção - manobra); intervenção (manobra); e pós-teste (pós-intervenção pós-manobra), desenvolvida em unidade de cuidados intermediários convencionais e unidade de terapia intensiva neonatal de maternidade escola, de atendimento terciário ao binômio mãe-filho, no Ceará-Brasil. A variável explanatória é inserção do PICC na 
veia basílica ou cefálica direita, puncionada na região cubital, que progrediu ou não, na região hemiclavicular direita de recém-nascidos. 0 desfecho esperado, nos casos em que o PICC não progrida, é a progressão deste em região hemiclavicular direitaapós aplicação da manobra e ponta do cateter em posicionamento central.

A amostra temporal constituiu de 64 inserções de PICC (inserido por punção direta, sem auxílio de ultrassom), em 58 recém-nascidos que atenderam aos critérios de inclusão, entre janeiro e abril de 2014, por quatro enfermeiras assistenciais das unidades, qualificadas, experientes e treinadas quanto ao método da pesquisa, procedimento operacional padrão da instituição e inserção de cateter. A cada procedimento, uma dupla de enfermeiras participava da inserção do PICC.

Incluíram-se recém-nascidos com indicação de PICC para infusão de antibióticos, nutrição parenteral, drogas vasoativas, em qualquer idade gestacional e peso ao nascer. Excluíram-se aqueles com malformação congênita, fraturas, luxações dos membros superiores e/ou clavículas, hematomas, equimoses no percurso braquial das veias basílica e cefálica direita, assim como a não realização da radiografia do tórax ou após seis horas da inserção do PICC.

$\mathrm{O}$ instrumento de coleta de dados constou de variáveis neonatais e perinatais, como sexo, peso ao nascer, idade gestacional (nascimento) e cronológica, diagnóstico médico de internação, indicação de implantação do cateter, escolha da veia pela randomização ou única veia puncionável, veia cateterizada, progressão do PICC, em região hemiclavicular direita, antes e após cada passo da manobra, e tempo decorrido da inserção e realização da radiografia do tórax.

O pré e pós-teste, necessários ao estudo quase experimental, foram caracterizados pela mensuração da progressão do cateter antes e depois da manobra da seguinte forma: com ombro abduzido a $90^{\circ}$ e cotovelo estendido, mensura-se em centímetros, a distância entre região cubital e região hemiclavicular direita (medida pré-teste), por meio de fita métrica colocada no ponto de inserção do cateter até o ponto hemiclavicular, também medido por meio da fita métrica; insere-se o cateter até a região hemiclavicular direita (obstáculo), aplica-se a manobra e inserem-se mais três centímetros do cateter (medida pós-teste), sendo esta a distância até o terceiro espaço intercostal (junção cavoatrial) em recém-nascido.

Para inserção dos recém-nascidos, respeitaram-se os critérios de inclusão, após assinatura do Termo de Consentimento Livre e Esclarecido pelos pais e/ou responsável. Após a decisão pela inserção do PICC (confeccionado em poliuretano, mono lúmen, 1,9 French), a dupla de enfermeiras treinadas realizou a avaliação da região cubital direita, visando observar a rede venosa e presença de lesões nesta área. A partir disso, decidiu-se pela forma de se escolher a veia puncionável. Definiu-se nesta pesquisa como veia puncionável aquelas visíveis e ou palpáveis, livre de obstáculos para punção, como estenose, presença de lesões prévias como hematomas e flebites, pois dificultam a punção, podendo acarretar injúrias ao recém-nascido.

Assim, quando as veias basílica e cefálica direita se encontravam puncionáveis, as enfermeiras responsáveis pela coleta sorteavam em qual veia se iniciava o procedimento ou quando existia apenas uma veia puncionável (basílica ou cefálica direita), escolhia-se a que estivesse disponível. Esse procedimento foi realizado após a eleição do recém-nascido para pesquisa e antes do início do procedimento.

Ao iniciar o procedimento, as duas enfermeiras decidiam quem faria a punção da veia e inserção do PICC. Feita a punção venosa, obtinham-se dois resultados: sucesso ou insucesso. Sendo a primeira punção bem-sucedida, o cateter era introduzido até a medida correspondente ao terceiro espaço intercostal direito. Se malsucedida, havendo outra veia puncionável, realizava-se a segunda punção, introduzindo o cateter até terceiro espaço intercostal direito, não havendo, encerrava-se a coleta.

Com punção bem-sucedida, inseria-se o cateter e se iniciava a inserção do cateter até região hemiclavicular direita. Com progressão nesta região, introdu- 
zia-se o cateter até junção cavoatrial e encerrava-se a coleta; sem progressão pela região hemiclavicular direita, aplicava-se a manobra. Ao realizar a manobra, obtinham-se dois resultados, progressão ou não progressão, após primeiro, segundo ou terceiro passo da manobra aplicada no ombro do recém-nascido. Com a progressão, avaliava-se, por radiografia do tórax, o posicionamento do cateter. Se posicionamento central, definia-se o resultado da manobra como totalmente bem-sucedida, no caso de posicionamento periférico, definia-se como manobra parcialmente bem-sucedida, ou seja, progrediu, porém, posicionou-se perifericamente.

Destaca-se que a verificação do posicionamento da ponta do cateter foi feita por meio da radiografia do tórax realizada até seis horas após término do procedimento. Caso ocorresse algum contratempo e a radiografia não fosse realizada, a inserção seria excluída da pesquisa, por saber-se da possibilidade de reposicionamento espontâneo do cateter, pós-inserção(7). No entanto, não houve exclusão de inserções, por esse motivo, no decorrer da pesquisa.

Os dados foram compilados no programa Excel versão Microsoft Office Excel 2010, processados e analisados no Programa Statistical Package for the Social Sciences 20.0. Para investigar se a progressão do cateter dependia do tipo de veia, foi considerado, primeiramente, o Teste Qui-Quadrado de Pearson. No entanto, devido ao elevado número de frequências esperadas menores que cinco (mais de $25,0 \%$ das células), utilizou-se do Teste Exato de Fisher com a mesma finalidade. A contribuição de cada manobra para mudança no alcance do cateter foi avaliada com auxílio do Teste de McNemar. Para possível relação entre a localização da ponta do PICC e o momento em que o cateter progrediu, empregou-se o Teste Qui-Quadrado de Pearson.

A pesquisa foi aprovada pelo Comitê de Ética e Pesquisa da instituição, conforme parecer no 408.041 e Certificado de Apresentação para Apreciação Ética no 22225113.5.0000.5050, e respeitaram-se as normas nacionais e internacionais de pesquisa envolvendo seres humanos.

\section{Resultados}

Participaram da pesquisa 58 recém-nascidos que atenderam aos critérios de inclusão, dos quais $53(91,4 \%), 4(6,9 \%)$ e $1(1,7 \%)$ foram submetidos a uma, duas e três inserções, respectivamente, totalizando amostra de 64 PICC inseridos. Das 64(100,0\%) inserções, 36(56,25\%) não progrediram e os recém-nascidos foram submetidos à manobra.

Observou-se predominância do sexo feminino, $30(51,7 \%)$, peso de nascimento 1.000 a 1.499 gramas $25(43,1 \%)$, idade gestacional ao nascer entre 30 e 34 semanas 30 (51,7\%). No momento da realização do PICC, a idade cronológica (dias) encontrou-se entre zero dia de vida e seis dias 49 (76,5\%). A prematuridade $53(91,4 \%)$ foi o diagnóstico médico de internação predominante.

Quanto à progressão do PICC, 27(42,2\%) ocorreram em veia basílica direita, dentre estas, $18(66,7 \%)$ eram única veia puncionável, $7(25,9 \%)$, devido ao sorteio da basílica, e 2(7,4\%) devido ao insucesso na punção da cefálica. Dentre $37(57,8 \%)$ inserções em veia cefálica, 17(46\%) foram a única puncionável e 15(40,5\%), resultado de sorteio da cefálica e 5(13,5\%), em virtude do insucesso na punção da basílica. Portanto, houve sucesso e, na primeira punção, em 57(89,06\%) inserções e 7(10,94\%), em segunda punção.

A Tabela 1 destaca frequência de progressão do cateter com e sem manobra pelas veias basílica e cefálica.

Tabela 1 - Distribuição das inserções dos cateteres em veia basílica e cefálica direita, com e sem aplicação da manobra de movimentação do ombro para progressão do cateter. Fortaleza, CE, Brasil, 2014

\begin{tabular}{|c|c|c|c|c|c|}
\hline \multirow[t]{2}{*}{ Veia } & $\begin{array}{c}\text { Progrediu } \\
\text { sem manobras }\end{array}$ & $\begin{array}{l}\text { Progrediu com } \\
\text { manobras }\end{array}$ & $\begin{array}{c}\text { Não } \\
\text { progrediu }\end{array}$ & Total & \multirow[t]{2}{*}{ p-valor* } \\
\hline & $n(\%)$ & $n(\%)$ & $n(\%)$ & $n(\%)$ & \\
\hline Basílica & $19(70,4)$ & $8(29,6)$ & - & $27(100,0)$ & \multirow{3}{*}{$<0,001$} \\
\hline Cefálica & $9(24,3)$ & $20(54,1)$ & $8(21,6)$ & $37(100,0)$ & \\
\hline Total & $28(43,8)$ & $28(43,8)$ & $8(12,4)$ & $64(100,0)$ & \\
\hline
\end{tabular}


A veia basílica mostrou-se mais favorável à progressão do PICC, pois 70,4\% das inserções progrediram sem manobra e 100,0\% após uso da manobra. Houve associação estatisticamente significante entre o uso da manobra e a progressão do PICC, especialmente quando a inserção foi realizada em veia cefálica, $24,3 \%$ das inserções progrediram sem manobra, $54,1 \%$ após manobra.

A Tabela 2 mostra a progressão do PICC, em veia basílica e cefálica direita de recém-nascidos, antes e após aplicação dos três passos da manobra.

Tabela 2 - Número de progressões de cateteres após aplicação dos passos da manobra de movimentação do ombro em recém-nascidos. Fortaleza, CE, Brasil, 2014

\begin{tabular}{lccccc}
\hline \multirow{2}{*}{ Passos } & $\begin{array}{c}\text { Antes da intervenção } \\
\text { Pré-teste }\end{array}$ & \multicolumn{2}{c}{$\begin{array}{c}\text { Pós intervenção } \\
\text { Pós-teste }\end{array}$} & \\
\cline { 2 - 5 } & $\begin{array}{c}\text { Não } \\
\text { progrediu }\end{array}$ & Progrediu & $\begin{array}{c}\text { Não } \\
\text { progrediu }\end{array}$ & Progrediu & p-valor* \\
\cline { 2 - 5 } & $\mathbf{n ( \% )}$ & $\mathbf{n ( \% )}$ & $\mathbf{n ( \% )}$ & $\mathbf{n ( \% )}$ & \\
\hline Primeiro & $36(100,0)$ & - & $21(58,3)$ & $15(41,7)$ & $<0,001$ \\
Segundo & $21(100,0)$ & - & $9(42,9)$ & $12(57,1)$ & $<0,001$ \\
Terceiro & $9(100,0)$ & - & $8(88,9)$ & $1(11,1)$ & $>0,999$ \\
\hline *Teste de McNemar (modelo binominal) & & & \\
\hline
\end{tabular}

A aplicação do primeiro e segundo passos da manobra resultou em mudança significativa na progressão dos cateteres. Uma vez aplicados os dois primeiros passos da manobra, a adição do terceiro passo não influenciou tanto a progressão dos PICC.

A Tabela 3 apresenta a distribuição das progressões dos cateteres, no primeiro e segundo passos da manobra e localização da ponta.

Apenas um cateter apresentou posicionamento periférico quando a progressão acontecia sem o uso de manobras. A distribuição do posicionamento do cateter após aplicar o primeiro passo da manobra $73,3 \%$ - 26,7\%) foi semelhante à composição observada após o segundo passo da manobra $(75,0 \%-25,0 \%)$.
Tabela 3 - Distribuição das progressões dos cateteres, observadas sem manobras e após primeiro e segundo passos da manobra e posicionamento da ponta do cateter. Fortaleza, CE, Brasil, 2014

\begin{tabular}{|c|c|c|c|}
\hline \multirow{3}{*}{ Etapas do procedimento } & \multicolumn{3}{|c|}{ Posicionamentos } \\
\hline & Central & Periférico & Total \\
\hline & n(\%) & n(\%) & n(\%) \\
\hline Sem manobras & $27(96,4)$ & $1(3,6)$ & $28(100,0)$ \\
\hline Após primeiro passo da manobra & $11(73,3)$ & $4(26,7)$ & $15(100,0) 0,032$ \\
\hline Após segundo passo da manobra & $9(75,0)$ & $3(25,0)$ & $12(100,0)$ \\
\hline Total & $47(85,5)$ & $8(14,5)$ & $55(100,0)$ \\
\hline
\end{tabular}

\section{Discussão}

Esta pesquisa apresenta como limitações: a ausência de cálculo amostral; a associação entre progressão do cateter e o material confeccionado, silicone e poliuretano; a aplicação da manobra no braço esquerdo; o emprego de cada passo da manobra separadamente; o pequeno número de recém-nascidos que receberam o terceiro passo da manobra; e o efeito da sequência dos passos da manobra sobre a progressão.

Pela análise estatística, percebeu-se o valor inestimável da manobra, devido favorecer a progressão do PICC em recém-nascidos pela veia basílica e cefálica direita, até veia cava, sendo de fácil aplicação, sem injúrias teciduais ou gastos adicionais. Tem-se como vantagem que a utilização da manobra pode evitar posicionamento inadequado, consequentemente, facilitar cumprimento da terapêutica endovenosa, contribuindo, assim, para qualidade de vida e promoção da saúde de recém-nascido internado.

Observou-se que a não progressão do cateter antes da manobra, pelas veias basílica e cefálica direita, na região hemiclavicular direita, ocorreu em mais da metade das cateterizações (56,3\%), sendo a cefálica a principal responsável pela não progressão $(75,7 \%)$. Tal resultado pode estar associado às carac- 
terísticas próprias do vaso, como a tortuosidade da veia ${ }^{(3)} \mathrm{e}$ angulação de inserção da veia cefálica em veia axilar, dificultando a progressão, o posicionamento inadequado e extravasamento ${ }^{(3)}$.

Em relação à veia basílica direita, favoreceu a progressão do PICC sem manobras, em 70,4\% das cateterizações. Enfatiza-se que essa veia é mais retilínea, calibrosa e curta, tem menor angulação de implantação na veia axilar e menor número de válvulas ${ }^{(3)}$.

Observou-se que apesar da veia basílica ter favorecido a progressão do PICC em 100,0\%, 29,6\% das inserções somente progrediram após o uso da manobra. Assim, o enfermeiro, ao ser capacitado e habilitado para inserção de PICC em recém-nascidos, sabendo aplicar a manobra, contribui para o aumento das chances de progressão e posicionamento central. É importante destacar o reconhecimento do papel do enfermeiro de acesso vascular na equipe de saúde, tendo a colaboração e o respeito interprofissional, influenciando positivamente na segurança do paciente ${ }^{(8)}$, além de que toda a equipe necessita estar preparada, a fim de evitar danos e possibilitar a permanência do cateter por maior tempo possível ${ }^{(9)}$.

Apesar da totalidade dos cateteres terem progredido em basílica após os três passos da manobra, enfatiza-se que dos cateteres que não progrediram, após o primeiro e segundo passos, a maioria ocorreu em veia cefálica e não progrediram após o terceiro passo da manobra e poucos em veia basílica. Infere-se, então, que o terceiro passo não contribuiu para progressão do PICC em veia cefálica. É pertinente examinar aspectos anatômicos da articulação do ombro, para melhor entendimento do efeito do terceiro passo.

Ao avaliar cada passo da manobra separadamente, aplicada em recém-nascidos em que o cateter não progrediu, houve semelhança entre quantidade de cateter que progrediu após o primeiro e segundo passos da manobra, em basílica e cefálica direita, com valor de $\mathrm{p}<0,001$. 0 fato de não ter-se aplicado unicamente o primeiro ou segundo passo da manobra, não foi avaliado se havia efeito cumulativo na progressão para cateteres que receberam o primeiro e, depois, o segundo passo.

Ao buscar associação entre os três passos da manobra e progressão do cateter em basílica e cefálica direita, verificou-se significância estatística, $p<0,001$, no primeiro e segundo passos, inferindo-se que estes contribuíram para o sucesso da manobra.

Nesta investigação, o desfecho parcialmente satisfatório da manobra refere-se ao posicionamento inadequado do cateter, ou seja, cateter que progrediu para posicionamento periférico (desfecho indesejado). Apesar da progressão do cateter na região hemiclavicular direita ter sido facilitada pela aplicação da manobra, é importante avaliar o posicionamento final da ponta do PICC, pela radiografia do tórax, observando progressão para veia cava superior ${ }^{(3)}$.

Ao se associar progressão sem manobra e após os dois primeiros passos da manobra e posicionamento central e periférico, encontrou-se significância estatística, para progressão sem manobra e posicionamento central, demonstrando que posicionamento periférico é raro, quando a progressão ocorre sem uso da manobra. A maioria dos cateteres inseridos progrediu para posicionamento central, após aplicação da manobra, evidenciando que a movimentação do ombro facilita a progressão do cateter em região hemiclavicular direita, pelas veias basílica e cefálica direita em recém-nascidos.

Apesar de a manobra haver viabilizado progressão da maioria dos cateteres, constatou-se posicionamento periférico em algumas inserções, ressaltando a importância de aprofundamento das pesquisas, para evitar posicionamento periférico e retirada não eletiva, por configurar-se clientela frágil e susceptível.

Pesquisa realizada com 563 inserções de PICC em recém-nascidos, em São Paulo, Brasil, para elaboração de modelo de regressão logística com fatores de risco para remoção não eletiva de PICC, verificou que o posicionamento não central da ponta apresentou duas vezes maior o risco de remoção não eletiva ${ }^{(10)}$.

Pesquisa desenvolvida com neonatos em Vitória, Brasil, mostrou que a localização da ponta do PICC foi central em $81(60,6 \%)$ das inserções ${ }^{(11)}$. Esses da- 
dos mostram a importância do posicionamento central do cateter, que viabiliza a infusão da terapêutica propostas, mitigando os riscos de injúrias teciduais, decorrentes da infusão de substâncias irritantes e/ou vesicantes em cateter posicionado perifericamente.

Há necessidade de aprofundamento de pesquisas, com vistas ao desenvolvimento de tecnologias capazes de solucionar o mau posicionamento do PICC em recém-nascidos e, consequentemente, mitigar potenciais complicações ${ }^{(12)}$, portanto, a manobra de movimentação do ombro torna-se uma dessas possibilidades.

Aponta-se a importância de se verificar o sucesso de cada passo da manobra na progressão do cateter. A elevação e protração do ombro facilitam a progressão do cateter em região hemiclavicular direita, pelas veias basílica e cefálica direita, em recém-nascidos, mostrando que a movimentação do ombro é uma estratégia viável no cuidado de Enfermagem ao neonato. E esse cuidado favorece as boas práticas com o PICC, contribuindo para segurança do paciente e qualidade do cuidado $^{(13)}$.

Ao enfocar o aspecto do posicionamento do cateter em neonatos, pesquisa retrospectiva de coorte, desenvolvida em unidades neonatais nos Estados Unidos, mostrou que a maioria dos cateteres estudados estavam em localização central no momento da inserção $(>90,0 \%)$, no entanto, não realizaram vigilância de rotina da posição da ponta, portanto, a migração da ponta não pode ser excluída ${ }^{(14)}$.

Ao final da pesquisa, decidiu-se intitular de Manobra de Elevação, Protração e Abaixamento do Ombro ou Manobra de EPA. A manobra foi criada a partir da prática profissional de uma enfermeira que presta assistência a recém-nascidos de alto risco, com 10 anos de experiência em Neonatologia. Recomenda-se que estudos sobre essa manobra sejam aprofundados e aplicados à Neonatologia, uma vez que esse posicionamento consiste em tecnologia de relevância científica, visando aprimoramento e incorporação na prática do ensino, pesquisa e cuidado a recém-nascidos, na perspectiva de prevenir complicações, promover a saúde e segurança de pacientes ${ }^{(15)}$.

\section{Conclusão}

A inserção de Cateter Central de Inserção Periférica em recém-nascidos está mais associada à progressão sem manobras pela veia basílica direita e progressão com manobras pela cefálica direita. Elevação e protração do ombro, em recém-nascidos, facilitou a progressão do cateter pela basílica e cefálica direita, sendo estatisticamente significantes. 0 primeiro e segundo passos da manobra (elevação e protração) favoreceram o posicionamento central.

\section{Colaborações}

Nobre KSS contribuiu na coleta, organização, interpretação dos dados, concepção do trabalho, redação e aprovação da versão final a ser publicada. Cardoso MVLML colaborou com delineamento, interpretação dos dados, redação e aprovação da versão final a ser publicada. Rodrigues EC e Melo GM auxiliaram na revisão crítica relevante do conteúdo intelectual e aprovação da versão final a ser publicada.

\section{Referências}

1. Unbeck M, Forberg U, Ygge BM, Ehrenberg A, Petzold $\mathrm{M}$, Johansson E. Peripheral venous catheter related complications are common among paediatric and neonatal patients. Acta Pædiatr. 2015; 104(6):566-74. doi: http://dx.doi.org/10.1111/ apa.12963

2. Telang N, Sharma D, Pratap OT, Kandraju H, Murki S. Use of real-time ultrasound for locating tip position in neonates undergoing peripherally inserted central catheter insertion: a pilot study. Indian J Med Res. 2017; 145(3):373-6. doi: http:// dx.doi.org/10.4103/ijmr.IJMR_1542_14

3. Doellman D, Buckner JK, Catudal JP, Frey AM, Lamagna $\mathrm{P}$, Runde DA, et al. Best practice guidelines in care and maintenance of pediatric central venous catheters. Ava Pediatric Special Interest Group. [Internet]. 2015 [cited 11 Dec, 2019]. Available from: http://hummingbirdmed. com/wp-content/uploads/AVA-GuidelinesPediatric-Guidelines.pdf 
4. Harada MJCS, Pedreira MLG. Terapia intravenosa e infusões. São Caetano do Sul (SP): Yendis; 2011.

5. Gandhi VG, Rali P, Shah P, Cheema T. Catheter pinch-off syndrome. Lung India [Internet]. 2017 [cited Jan 11, 2020]; 34(5):470-1. Available from: http://www.lungindia.com/text. asp?2017/34/5/470/213825

6. Sharpe E, Kuhn L, Ratz D, Krein SL, Chopra V. Neonatal peripherally inserted central catheter practices and providers. Adv Neonatal Care. 2017; 17(3):209-21. doi: http://dx.doi.org/10.1097/ ANC.0000000000000376

7. Beccaria P, Silvetti S, Mucci M, Battini I, Brambilla P, Zangrillo A. Contributing factors for a late spontaneous peripherally inserted central catheter migration: a case report and review of literature. J Vasc Access; 2015; 16(3):178-82. doi: http:// dx.doi.org/10.5301/jva.5000337

8. Krein SL, Kuhn L, Ratz D, Winter S, Vaughn VM, Chopra V. The relationship between perceived role and appropriate use of peripherally inserted central catheters: a survey of vascular access nurses in the United States. Int J Nurs Stud. 2017; 71:28-33. doi: http://dx.doi.org/10.1016/j. ijnurstu.2017.03.001

9. Bortoli PS, Leite AC, Alvarenga WA, Alvarenga CS, Bessa CR, Nascimento LC. Peripherally inserted central catheter in pediatric oncology: a scoping review. Acta Paul Enferm. 2019; 32(2):220-8. doi: dx.doi.org/10.1590/1982-0194201900030
10. Costa P, Kimura AF, Brandon DH, Paiva ED, Camargo PP. The development of a risk score for unplanned removal of peripherally inserted central catheter in newborns. Rev Latino-Am Enfermagem. 2015; 23(3):475-82. doi: http:// dx.doi.org/10.1590/0104-1169.0491.2578

11. Rangel RJM, Castro DS, Amorim MHC, Zandonade E, Christoffel MM, Primo CC. Practice of insertion, maintenance and removal of peripheral inserted central catheter in neonates. Rev Pesq Fundament Care online. 2019; 11(n.esp):278-84. doi: http:// dx.doi.org/10.9789/2175-5361.2019.v11i2.278284

12. Franklin I, Gilmore C. Placement of a peripherally inserted central catheter into the azygous vein. J Med Radiat Sci. 2015; 62(2):160-2. doi: http:// dx.doi.org/10.1002/jmrs.98

13. Nobre KSS, Cardoso MVLML, Teixeira JL, Lopes MMCO, Fontenele FC. Use of peripherally inserted central catheter in a neonatal unit: a descriptive study. Online Braz J Nurs. 2016; 15(2):215-25. doi: dx.doi.org/10.17665/1676-4285.20165420

14. Beard L, Levek C, Hwang S, Grover T. Prediction of nonelective central venous catheter removal in medically complex neonates. Pediatr Qual Saf. 2019; 4(4):e179. doi: http://dx.doi.org/10.1097/ pq9.0000000000000179

15. Manzo BF. Nursing performance in strengthening patient safety in neonatology. Rev Enferm CentroOeste Min. 2018; 8:eEditorial. doi: http://dx.doi. org/10.19175/recom.v8i0.3226

\section{(cc) BY}

Este é um artigo de acesso aberto distribuído sob os termos da Licença Creative Commons 